\title{
UTILIZAÇÃO DO ÂNION GAP URINÁRIO NO DIAGNÓSTICO DE ACIDOSE TUBULAR RENAL
}

\author{
Urinary anion gap use on diagnosis of renal tubular acidosis
}

\author{
Julio Cesar da Costa Marnatti ${ }^{1}$, Adroaldo Lunardelli ${ }^{2}$
}

\section{6) ACESSO LIVRE}

Citação: Marnatti JCC, Lunardelli A. (2020) Utilização do ânion gap urinário no diagnóstico de acidose tubular renal. Revista de Patologia do Tocantins, $7(1): 27-32$

\begin{abstract}
Instituição: $\quad$ 'Biomédico.Centro Universitário Ritter dos Reis (UniRitter). Porto Alegre, Rio Grande do Sul, Brasil. ${ }^{2}$ Farmacêutico Doutor. Professor do Centro Universitário Ritter dos Reis (UniRitter). Porto Alegre, Rio Grande do Sul, Brasil.
\end{abstract}

Autor correspondente: Prof. Dr. Adroaldo Lunardelli, Laboratório de Análises Clínicas do Centro Universitário Ritter dos Reis (UniRitter), Rua Orfanotrófio 555, bloco C, segundo andar, Porto Alegre, Rio Grande do Sul, Brasil. CEP 90840-440. Telefone: 32303333.

E-mail:

adroaldolunardelli@gmail.com

Editor: Guedes V. R. Medicina, Universidade Federal do Tocantins, Brasil.

Publicado: 28 de junho de 2020.

Direitos Autorais: (c) 2020 Marnatti et al. Este é um artigo de acesso aberto que permite o uso, a distribuição e a reprodução sem restrições em qualquer meio, desde que o autor original e a fonte sejam creditados.Conflito de interesses: os autores declararam que não existem conflitos de interesses.

\begin{abstract}
RESUMO
Introdução:A acidose tubular renal (ATR) é uma doença obscura e de difícil diagnóstico, pois causa uma alteração no balanço acidobásico do organismo. Devido à complexidade dos mecanismos fisiológicos que controlam o pH plasmático e urinário, podendo ser de origem congênita ou adquirida, com sintomas que variam conforme o tipo de acidose presente e acarretam num diagnóstico complexo e muitas vezes demorado. Objetivo: Revisar a literatura quanto a utilização e a importância do ânion gap urinário (AGU) como ferramenta no diagnóstico das ATRs e proporcionar um método investigativo para facilitar a identificação da acidose renal. Materiais e métodos: Trata-se de uma revisão integrativa da literatura onde foram acessadas as bases de dados Medline/Pubmed, Science Direct e Scielo. Utilizou-se os descritores: acidose, tubular, renal e ânion gap urinário, bem como a combinação destas palavras. Resultados: Dos 151 artigos encontrados, foram identificados 6 estudos que utilizam o ânion gap urinário demonstrando o seu potencial como ferramenta diferencial no diagnóstico da acidose tubular renal. Conclusão: O AGU consegue confirmar rapidamente a presença da ATR eliminando a possibilidade de perda extrarrenal do bicarbonato, como problemas gastrointestinais ou em pacientes com acidose metabólica hiperclorêmica. O AGU é uma ferramenta de grande valia diagnóstica para as ATRs, já que necessita de informações de fácil acesso ou previamente obtidas, apresenta um baixo custo e há possibilidade de diagnóstico exato, desde que se leve em conta suas limitações.
\end{abstract}

Palavras-chave: Acidose tubular renal; bicarbonato; nefropatia.

\begin{abstract}
Introduction: Renal tubular acidosis is an obscure disease with a hard diagnosis that causes changes to the acid-balance of the organism. Due the complexity of the physiological mechanisms that control both the plasma and urinary $\mathrm{pH}$, which may be of congenital or acquired origin and symptoms that vary according to the type of acidosis present which leads to a complex and often time-consuming diagnosis. Objective: The aim of this study was to review the literature on the use and importance of urinary anion gap as a tool in the diagnosis of RTAs and to provide an investigative method to streamline the identification of renal acidosis. Materials and Methods: It is an integrative review of the literature where the Medline/Pubmed, Science Direct and Scielo databases were accessed. The following descriptors were used: renal, tubular, acidosis, urinary anion gap, and were used as well as the combination of these words. Results: From the 151 articles found, 6 studies were identified that use the urinary anion gap demonstrating its potential as a differential tool in the diagnosis of renal tubular acidosis. Conclusion: The AGU can confirm the presence of ATR rapidly eliminating the possibility of extrarenal loss of bicarbonate, such as gastrointestinal problems or in patients with hyperchloremic metabolic acidosis. The AGU is a highly diagnostic tool for the ATRs, since it requires information that is easily accessible or previously obtained, presents a low cost and there is possibility of accurate diagnosis, as long as its limitations are taken into account.
\end{abstract}

Keywords: Renal tubular acidosis; bicarbonate; kidney diseases. 


\section{INTRODUÇÃO}

Segundo dados de 2015 da National Kidney Foundation ${ }^{1}$, 10\% da população mundial sofre de alguma doença renal crônica (DRC) e, em 2010, estima-se que 2,3 a 7,1 milhões de pessoas sucumbiram por doenças renais em estágio final e outras 1,7 milhões morreram por complicações originadas da insuficiência renal aguda, com um total de 5 a 10 milhões de pessoas falecendo, anualmente, devido à complicações renais. Em 2010, mais de 2,62 milhões pessoas, no mundo, realizaram tratamentos como hemodiálise ou transplante renal para permanecerem vivas, com a projeção de que esse número dobre até $2030^{2}$. Acredita-se, todavia, que esse número represente apenas $10 \%$ da população que necessita de algum tipo de intervenção para sobreviver e, daqueles que estão sendo tratados, a maioria se encontra em apenas cinco países: Alemanha, Brasil, Estados Unidos, Itália e Japão, sendo que estas comarcas somam apenas $12 \%$ da população mundial ${ }^{12}$. Conforme Lozano et al. $^{3}$ as doenças renais em 1990 ocupavam a 27 a posição no ranking mundial de causas de mortes no mundo e, em 2010, passaram a ocupar 18a posição, crescimento que só havia sido observado anteriormente pelo vírus do HIV. Acredita-se que esses números continuem subindo conforme a população de países em desenvolvimento envelheça, bem como pela falta de instrução popular acerca das complicações renais e o difícil acesso a dados epidemiológicos. Doenças renais possuem alto custo associado aos seus tratamentos, forçando países desenvolvidos gastarem de 2 a $3 \%$ do seu orçamento na área da saúde no tratamento de insuficiência renal, mesmo que aqueles que recebem o tratamento representem menos de $0,03 \%$ da população ${ }^{1}$.

Os rins possuem um importante papel no equilíbrio acidobásico do organismo, uma vez que são responsáveis pela reabsorção do bicarbonato $\left(\mathrm{HCO}_{3}^{-}\right)$na porção proximal dos túbulos renais e pela secreção de ácidos, principalmente o amônio $\left(\mathrm{NH}_{4}^{+}\right)$na porção distal dos ductos ${ }^{4}$. Defeitos nesses mecanismos levam a um descontrole do potencial hidrogeniônico $(\mathrm{pH})$ urinário, que podem ocasionar acidose tubular renal (ATR). Essa nomenclatura é utilizada para descrever um grupo de doenças renais raras que são caracterizadas por desequilíbrio na regulação acidobásica que podem ser de origem hereditária ou adquirida $^{5}$. As ATRs apresentam alterações iônicas com presença de acidose metabólica sem grandes alterações nas funções renais, mantendo a taxa de filtração glomerular (TFG) normalizada, com o distúrbio tubular sendo responsável pelo desequilíbrio do $\mathrm{pH}$ urinário ${ }^{6}$. As ATRs estão classificadas em:

ATR distal (ATR I) - É associada com a redução da excreção de íons de hidrogênio $\left(\mathrm{H}^{+}\right)$na porção distal do néfron resultando em $\mathrm{pH}$ urinário persistentemente elevado (superior a 5,5) e acidose sistêmica?.

ATR proximal (ATR II) - Ocorre defeito na reabsorção de $\mathrm{HCO}_{3}$ na porção proximal dos túbulos renais tornando o $\mathrm{pH}$ urinário superior a 6,0. Grande parte dos casos de ATR proximal ocorrem devido à disfunção generalizada dos túbulos conhecida como síndrome de Fanconi ${ }^{8}$.
ATR proximal e distal (ATR III) - Apresentando propriedades das ATRs I e II, é extremamente rara e se desenvolve devido à mutação no gene da anidrase carbônica tipo II, que altera a produção e reabsorção do bicarbonato urinário9.

ATR hipercalêmica (ATR IV) - Sua principal causa é o hipoaldosteronismo, que ocasiona redução da síntese, liberação e secreção de aldosterona ou devido à resistência do órgão alvo. A aldosterona também medeia a reabsorção dos íons sódio $\left(\mathrm{Na}^{+}\right)$em troca do íon potássio $\left(\mathrm{K}^{+}\right)$e do íon hidrogênio $\left(\mathrm{H}^{+}\right)$via antiporter; deste modo ocorre redução da excreção de potássio, levando à hipercalemia seguida da redução na excreta de ácidos pelos rins ${ }^{10}$.

Apesar das ATRs serem pouco reconhecidas e com quase nenhum dado epidemiológico disponível, elas contribuem para as estatísticas de DRCs, pois estão presentes em pacientes em estado crítico que muitas vezes já apresentam - ou estão evoluindo - para a insuficiência renal ${ }^{11}$. É importante ressaltar que, apesar do conhecimento das acidoses há muitos anos, ainda existe grande dificuldade em chegar a um diagnóstico que é, por muitas vezes, complexo e demorado ${ }^{12}$.

Uma importante ferramenta no diagnóstico dessas ATRs é o cálculo do ânion gap urinário (AGU), pois ele proporciona um valor estimado dos níveis de excreção do $\mathrm{NH}_{4}{ }^{+}$através da soma dos cátions $\mathrm{Na}^{+}$e $\mathrm{K}^{+}$subtraído do principal ânion, o cloreto $\left(\mathrm{Cl}^{-}\right.$ )$^{13}$. O ânion gap urinário é calculado através da equação:

Ânion gap urinário $=\left(\left[\mathrm{Na}^{+}\right]+\left[\mathrm{K}^{+}\right]\right)-\left[\mathrm{Cl}^{-}\right]$

As ATRs necessitam ser diagnosticadas de maneira exata e rápida para o tratamento eficaz, visto que é possível desenvolver sintomas gravíssimos, principalmente em crianças. Deve-se formar uma linha de investigativa concisa e eficaz através de testes de curta duração e que consigam eliminar hipóteses. O intento deste desígnio é, portanto, investigar o papel do AGU como opção diagnóstica das ATRs.

\section{METODOLOGIA}

O presente estudo consiste em uma revisão integrativa da literatura com base na busca de artigos científicos disponíveis em diferentes bancos de dados. A busca foi realizada entre os meses de janeiro e junho de 2019 nas bases de dados Medline/Pubmed, Science Direct e Scielo, nos idiomas português e inglês, abrangendo artigos publicados entre 2009 e 2019. Utilizou-se os descritores: acidose, tubular, renal, ânion gap urinário e as suas devidas combinações, bem como estes unitermos na língua inglesa (renal, tubular, acidosis, urinary ânion gap). Foi adotado como critério de inclusão as publicações cuja temática abordara os resultados da utilização do hiato aniônico urinário para o diagnóstico de acidose tubular renal e/ou que demonstravam a importância dele na investigação das acidoses. Primeiramente, foram analisados títulos e resumos dos resultados das buscas, sendo selecionados aqueles estudos que contemplaram a temática 
proposta, incluídos e lidos de forma detalhada. Foram excluídos da seleção os estudos realizados em animais. A análise seguiu referencial teórico, incluindo a leitura exploratória, seletiva, analítica e interpretativa do material obtido.

\section{RESULTADOS E DISCUSSÃO}

Através das buscas realizadas e de acordo com os critérios descritos anteriormente, foram encontrados 151 estudos (32 artigos na base de dados Science Direct, 126 na base de dados Medline/Pubmed e 3 na base de dados Scielo). Deste total, 89 estudos foram excluídos por restrição de adequação, restando 61 artigos potencialmente elegíveis. Dentre estes, 6 trabalhos contemplaram os critérios de inclusão do presente estudo e são descritos na Tabela 1.

\begin{tabular}{lll}
\hline Autores & Periódico $e$ ano & Ano \\
\hline Alexander RT et al. ${ }^{16}$ & Pediatric Clinics of North America & 2019 \\
\hline Batlle D et al. ${ }^{36}$ & Clinical Journal of the American Society of Nephrology & 2018 \\
\hline Muthukrishnan J et al. ${ }^{34}$ & Medical Journal Armed Forces India & 2015 \\
\hline Sarma A ${ }^{32}$ & Indian Journal of Endocrinology and Metabolism & 2018 \\
\hline Soleimani M et al. ${ }^{31}$ & American Journal of Kidney Diseases & 2016 \\
\hline Yaxley J et al.30 & Ochsner Journal & 2016 \\
\hline
\end{tabular}

Tabela 1 - Trabalhos selecionados e analisados no presente estudo com respectivos autores, periódico e ano de publicação

É necessário o conhecimento básico dos mecanismos fisiológicos que participam do balanço acidobásico e quais alterações são geradas quando deixam de exercer suas funções pelos diferentes tipos de $\mathrm{ATRs}^{14}$. Um dos fatores que atrapalham no seu diagnóstico é a sua representação clínica, que pode ser completamente assintomática até situações mais complexas com cálculos renais, nefrocalcinose e quadros de hipocalemia tão severos que levam à morte. Seu prognóstico é semelhante à sua representação clínica, pois alguns pacientes conseguem se manter com um tratamento mínimo, e outros evoluem à insuficiência renal em estágio terminal. Nestes casos, as crianças são as mais afetadas, tendo seu desenvolvimento físico e, possivelmente, psicológico prejudicados porque geralmente desenvolvem a doença por causas primárias, desse modo, sofrem com as ATRs durante a fase de crescimento ${ }^{15}$. Crianças com ATR podem apresentar baixo crescimento, depleção de volume, fadiga ou letargia ${ }^{16}$. As causas e sintomas mais relevantes são relatadas na Tabela 2.

\begin{tabular}{|c|c|c|c|}
\hline $\begin{array}{l}\text { Tipos de } \\
\text { acidoses }\end{array}$ & $\begin{array}{l}\text { Acidose tubular distal ou ATR } \\
\text { tipo I }\end{array}$ & $\begin{array}{l}\text { Acidose tubular } \\
\text { proximal ou ATR tipo II }\end{array}$ & $\begin{array}{c}\text { Acidose tubular } \\
\text { hipercalêmica ou ATR } \\
\text { tipo IV }\end{array}$ \\
\hline $\begin{array}{c}\text { Causas } \\
\text { primárias }\end{array}$ & $\begin{array}{l}\text { Mutações hereditárias ou } \\
\text { somáticas (ATP6V1P1, entre } \\
\text { outras). }\end{array}$ & $\begin{array}{l}\text { Mutações hereditárias ou } \\
\text { somáticas (SLC4A4, entre } \\
\text { outras). }\end{array}$ & $\begin{array}{l}\text { Mutações hereditárias ou } \\
\text { somáticas (WNK4, entre } \\
\text { outras). }\end{array}$ \\
\hline $\begin{array}{c}\text { Causas } \\
\text { secundárias }\end{array}$ & $\begin{array}{c}\text { Síndrome de Sjögren, lúpus } \\
\text { eritematoso sistêmico, hepatites, } \\
\text { cirrose biliar primária, } \\
\text { nefrotoxinas, artrite reumatoide, } \\
\text { medicamentos. }\end{array}$ & $\begin{array}{c}\text { Síndrome de Fanconi, } \\
\text { síndrome de Sjögren, } \\
\text { nefrotoxinas, síndrome } \\
\text { metabólica, } \\
\text { medicamentos. }\end{array}$ & $\begin{array}{c}\text { Supressão adrenal, } \\
\text { hipertensão, resistência à } \\
\text { aldosterona, inibidores de } \\
\text { angiotensina, DRCs, } \\
\text { medicamentos. }\end{array}$ \\
\hline $\begin{array}{c}\text { Sintomas e } \\
\text { consequências }\end{array}$ & $\begin{array}{l}\text { Raquitismo, problemas de } \\
\text { crescimento, osteomalácia, } \\
\text { surdez, nefrocalcinose, } \\
\text { enfraquecimento muscular e } \\
\text { arritmia. }\end{array}$ & $\begin{array}{l}\text { Osteomalácia, raquitismo, } \\
\text { problemas de } \\
\text { crescimento, letargia, } \\
\text { respiração alterada. }\end{array}$ & $\begin{array}{l}\text { Hipotensão postural, } \\
\text { letargia, nefrolitíase, variam } \\
\text { conforme a DRC presente. }\end{array}$ \\
\hline
\end{tabular}

Tabela 2 - Causas e sintomas mais relevantes para cada tipo de acidose tubular.

Acidose tubular distal: A porção distal dos túbulos é formada $40 \%$ de células intercaladas alfa responsáveis pela acidificação e transporte ativo de $\mathrm{H}^{+}$para o lúmen do vaso, os outros $60 \%$ são formados de células principais que contêm bombas de sódio e potássio voltadas para o espaço intersticial e canais dos mesmos íons para o lúmen. Essa combinação de proteínas ocasiona um gradiente negativo no interior do túbulo facilitando a acidificação da urina pelas células intercaladas alfa ${ }^{17}$. Existem duas variações mais relevantes de ATR tipo 1: a clássica (que é um defeito nas células intercaladas alfa, impedindo a secreção de hidrogênio) e a denominada voltagem dependente (que é uma falha nos canais iônicos, logo, o gradiente tubular se altera dificultando o transporte de $\left.\mathrm{H}^{+}\right)^{18}$.

Acidose tubular proximal: $O$ túbulo proximal é responsável por 90\% do bicarbonato filtrado diariamente ser reabsorvido, sendo que não há uma proteína transportadora de $\mathrm{HCO}_{3}{ }^{-}$, e que enquanto no lúmen o bicarbonato de sódio $\left(\mathrm{NaHCO}_{3}\right)$ está ionizado, as células presentes na porção proximal apresentam proteínas antiporter de sódio/hidrogênio e anidrase carbônica. Desse modo, o hidrogênio chega no lúmen, se liga no $\mathrm{NaHCO}_{3}$ formando ácido carbônico $\left(\mathrm{H}_{2} \mathrm{CO}_{3}\right)$, que será decomposto pela anidrase carbônica em gás carbônico $\left(\mathrm{CO}_{2}\right)$ e água $\left(\mathrm{H}_{2} \mathrm{O}\right)$, moléculas de fácil difusão que entram na célula para o processo que ocorreu no lúmen ser revertido, portanto $\mathrm{O}^{\mathrm{CO}_{2}}$ será hidratado formando novamente $\mathrm{H}_{2} \mathrm{CO}_{3}$ que em seguida será retirado um $\mathrm{H}^{+}$, o qual retornará para o lúmen. ${\mathrm{O} \mathrm{HCO}_{3}}^{-}$ formado por esse processo será reabsorvido por cotransportadores (proteína simporter) de $\mathrm{HCO}_{3}^{-}$e $\mathrm{K}^{+}$, sendo que qualquer defeito no mecanismo descrito ocasionará a perda de $\mathrm{HCO}_{3}{ }^{-}$e, por conseguinte, aumento no $\mathrm{pH}$ urinário ${ }^{19}$.

Acidose tubular hipercalêmica: A ATR tipo IV apresenta propriedades semelhantes à ATR distal, porém com uma hipercalemia característica que pode ser causada por deficiência ou resistência à aldosterona, assim, ocorre diminuição na secreção de $\mathrm{H}^{+}$e $\mathrm{K}^{+}$e, consequentemente, elevação do $\mathrm{pH}$ urinário e prejuizo no funcionamento dos canais de sódio e potássio, com a hipercalemia reduzindo a amoniogênese ${ }^{20}$.

A principal característica da acidose tubular renal é a presença de acidose metabólica de ânion gap normal. Essa particularidade é a principal motivação da busca por uma acidose tubular renal. No cálculo do ânion gap (AG) é feito a diferença dos principais cátions $\left(\mathrm{Na}^{+} \mathrm{e} \mathrm{K}^{+}\right)$pelos principais ânions $\left(\mathrm{Cl}^{-}\right.$e $\left.\mathrm{HCO}_{3}{ }^{-}\right)$presentes no plasma, formando a equação demonstrada na Tabela 3.

\begin{tabular}{|c|c|c|}
\hline Nome da equação & Equação & $\begin{array}{l}\text { Valor de } \\
\text { referência }\end{array}$ \\
\hline $\begin{array}{l}\text { Ânion gap } \\
\text { (AG) }\end{array}$ & $\left(\left[\mathrm{Na}^{+}\right]+\left[\mathrm{K}^{+}\right]\right)-\left([\mathrm{Cl}]+\left[\mathrm{HCO}_{3}\right]\right)$ & $8-16 \mathrm{mEq} / \mathrm{L}$ \\
\hline $\begin{array}{l}\text { Ânion gap urinário } \\
\text { (AGU) }\end{array}$ & $\left(\left[\mathrm{Na}^{+}\right]+\left[\mathrm{K}^{+}\right]\right)-[\mathrm{Cl}]$ & $20-90 \mathrm{mEq} / \mathrm{L}$ \\
\hline $\begin{array}{l}\text { Fração excretada } \\
\text { de bicarbonato } \\
\left(\mathrm{FeHCO}_{3}^{-}\right)\end{array}$ & $\begin{array}{c}{\left[\left(\left[\mathrm{HCO}_{3}^{-}\right] \text {urinário } X \text { [creatinina] plasmática }\right) /\left(\left[\mathrm{HCO}_{3}^{-}\right]\right.\right.} \\
\text {plasmático } X \text { [creatinina] urinária) }] \times 100\end{array}$ & $5-10 \%$ \\
\hline $\begin{array}{l}\text { Gap osmolar } \\
\text { urinário }\end{array}$ & $2 \mathrm{X}\left(\left[\mathrm{Na}^{+}\right]+\left[\mathrm{K}^{+}\right]\right)+[$ureia $] / 6+[$ glicose $] / 18$ & $\begin{array}{c}80-150 \\
\mathrm{mEq} / \mathrm{L}\end{array}$ \\
\hline $\begin{array}{c}\text { Gradiente de } \\
\text { potássio } \\
\text { transtubular } \\
\text { (TTKG) }\end{array}$ & 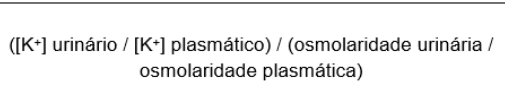 & $8-9 \mathrm{mEq} / \mathrm{L}$ \\
\hline
\end{tabular}


Tabela 3 - Equações utilizadas no tangente ao diagnóstico de acidose tubular renal.

Um resultado elevado, em acidose metabólica, significa retenção de ácidos que não são compensados pelo $\mathrm{Cl}^{\prime}$, como no caso de cetoacidoses. Já um AG normal (dentro do valor de referência) indica queda no bicarbonato e aumento do cloreto plasmático. Essa representação se deve à perda gastrointestinal de $\mathrm{HCO}_{3}{ }^{-}$ou redução na reabsorção de bicarbonato pelos rins, que também pode acarretar numa excreção menor de amônia. É preciso considerar que o resultado do $A G$ não leva em conta ânions não mensurados, como albumina e fosfato ${ }^{21-22}$. Após a confirmação de que não há perda gastrointestinal de bicarbonato, é crucial que seja checado a medicação do paciente, pois como demonstrado por Shiber ${ }^{23}$, em 2010, e Riveiro-Barciela et al. ${ }^{24}$, em 2011, medicamentos como o topiramato (inibidor da anidrase carbônica) e o tacrolimus (imunossupresor que desativa as bombas de $\mathrm{Na}^{+} / \mathrm{K}^{+}$) modificam o funcionamento de determinadas proteínas envolvidas no controle iônico. Caso haja presença de tais medicamentos recomenda-se a redução da dosagem ou troca da medicação e, em seguida, repetir os testes anteriores (Figura 1).

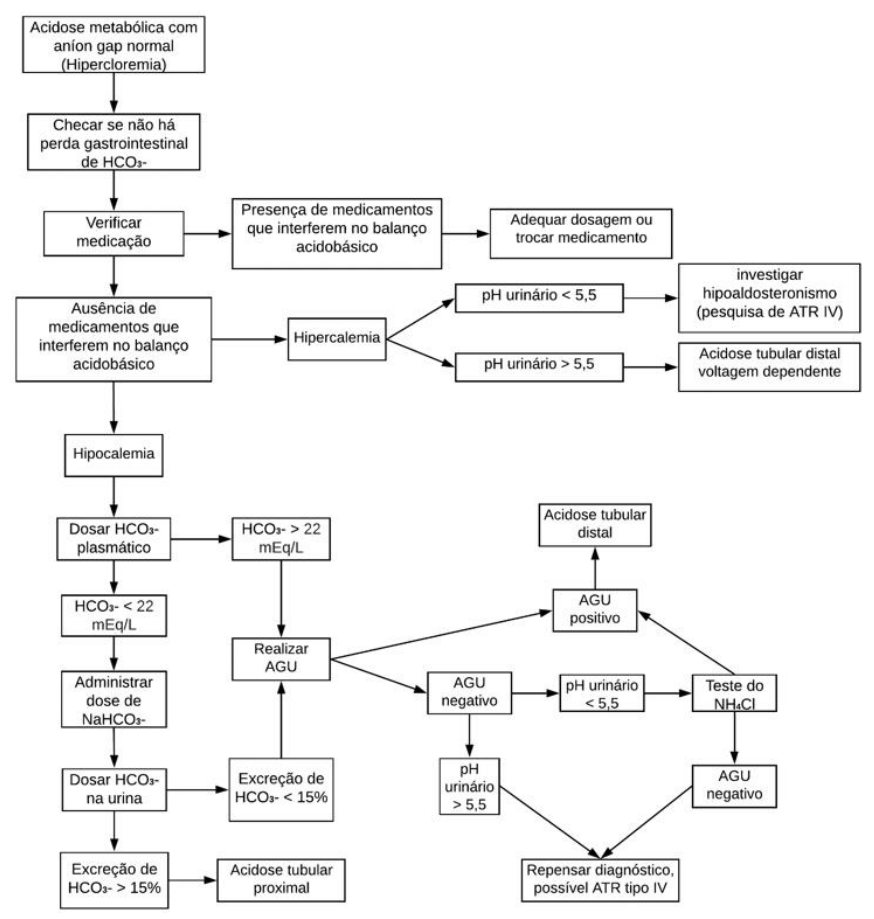

Figura 1 - Fluxograma de acordo com a lógica usada na investigação das ATRs. pH: potencial hidrogeniônico; $\mathrm{HCO}_{3}{ }^{-}$: bicarbonato; $\mathrm{AGU}$ : ânion gap urinário; >: superior $\mathrm{a}$; <: inferior $\mathrm{a}$.

Assim, casos de hipercalemia e hipocalemia são vitais para o diagnóstico correto, dado que em condições de hipercalemia a suspeita é da presença de ATR tipo IV, com a maioria dos casos ocorrendo por hipoaldosteronismo primário ou secundário. Também é possível, entretanto, que se trate de um caso mais raro como ATR distal voltagem dependente, essa diferenciação é feita pela titulação do $\mathrm{pH}$ urinário como visto na Tabela 4.

\begin{tabular}{|c|c|c|c|c|c|c|c|c|}
\hline $\begin{array}{l}\text { Tipos de } \\
\text { acidoses }\end{array}$ & $\begin{array}{c}\mathrm{pH} \\
\text { plasmático }\end{array}$ & $\begin{array}{c}\mathrm{pH} \\
\text { urinário }\end{array}$ & $\begin{array}{c}{\left[K^{+}\right]} \\
\text {sérico } \\
\text { (mEq/L) }\end{array}$ & $\begin{array}{l}{\left[\mathrm{HCO}_{3}\right]} \\
\text { sérico } \\
\text { (mEq/L) }\end{array}$ & $\begin{array}{c}{\left[\mathrm{Na}^{+}\right]} \\
\text {urinário } \\
\text { (mEq/L) }\end{array}$ & $\begin{array}{c}\text { AGU } \\
\text { (mEq/L) }\end{array}$ & [Aldosterona] & [Amônia] \\
\hline $\begin{array}{c}\text { Acidose tubular } \\
\text { distal ou ATR } \\
\text { tipo I }\end{array}$ & $<7,35$ & $>5,5$ & $<3,6$ & $<10-20$ & $40-200$ & $>0$ & Normal & Baixa \\
\hline $\begin{array}{l}\text { Acidose tubular } \\
\text { distal voltagem } \\
\text { dependente }\end{array}$ & $<7,35$ & $>5,5$ & $>5,2$ & $<10-20$ & $>150$ & $>0$ & $\begin{array}{l}\text { Levemente } \\
\text { elevado }\end{array}$ & Baixa \\
\hline $\begin{array}{l}\text { Acidose tubular } \\
\text { proximal ou } \\
\text { ATR tipo II }\end{array}$ & $<7,35$ & $\begin{array}{c}>5,5 \text { ou } \\
<5,5 \text { se } \\
\text { o HCO3- } \\
\text { estiver } \\
\text { abaixo } \\
\text { do limiar } \\
\text { renal }\end{array}$ & $<3,6$ & $12-18$ & $\begin{array}{c}\text { Não } \\
\text { mensurado }\end{array}$ & $\begin{array}{c}\text { Não } \\
\text { mensurado }\end{array}$ & Normal & Normal \\
\hline $\begin{array}{l}\text { Acidose tubular } \\
\text { hipercalêmica } \\
\text { ou ATR tipo IV }\end{array}$ & $<7,35$ & $<5,5$ & $>5,2$ & $>17$ & $\begin{array}{l}\text { Elevado } \\
\text { mesmo em } \\
\text { dietas de } \\
\text { restriçäo }\end{array}$ & $<0$ & $\begin{array}{l}\text { Baixo ou } \\
\text { elevado (varia } \\
\text { conforme o } \\
\text { motivo do } \\
\text { hipoaldoste- } \\
\text { ronismo) }\end{array}$ & $\begin{array}{l}\text { Normal ou } \\
\text { abaixo }\end{array}$ \\
\hline
\end{tabular}

$>$ : superior a; < inferior a.

Tabela 4 - Comparação dos principais elementos alterados pelos diferentes tipos de ATRs

Outra opção, além do pH urinário, é o gradiente de potássio transtubular (TTKG) com um resultado inferior a $4 \mathrm{mEq} / \mathrm{L}$ indicando problemas no transporte do $\mathrm{K}^{+}$e confirmando a ATR voltagem dependente ${ }^{25-27}$. Em estados de hipocalemia é de extrema importância a dosagem do $\mathrm{HCO}_{3}{ }^{-}$sérico; se o resultado for normal, o próximo passo deve ser a realização do AGU; porém, quando abaixo do limiar renal, é recomendado medir o clearence do bicarbonato. Esse exame é o padrão ouro para confirmação de ATR tipo II e consiste em infundir uma solução de bicarbonato de sódio e titular $\mathrm{O}_{\mathrm{HCO}_{3}}^{-}$urinário acompanhando ao mesmo tempo seus níveis séricos, pois quando ultrapassado o limiar renal, o bicarbonato volta a ser filtrado e, portanto, se a excreção do bicarbonato ultrapassar $15 \%$ consegue-se comprovar a acidose tubular proximal. Outra opção para o mesmo teste é fazer um comparativo da fração excretada de bicarbonato (demonstrada na Tabela 3) de hora em hora até os níveis de $\mathrm{HCO}_{3}{ }^{-}$normalizarem: apresentando $\mathrm{pH}$ superior a 7,5 ou excreção maior que $15 \%$, é confirmatório para ATR tipo II; se em qualquer um dos métodos o resultado for inferior a $15 \%$, deve-se prosseguir com o uso do $\mathrm{AGU}^{28-29}$.

Os estudos realizados por Yaxley et al. ${ }^{30}$ e Soleimani et al. ${ }^{31}$ corroboram que o AGU é uma etapa imprescindível no diagnóstico de ATRs tipo I e IV, pois ele serve como um marcador substituto à titulação do ânion $\mathrm{NH}_{4}^{+}$agilizando o diagnóstico. Dessa forma, um AGU positivo após o teste do bicarbonato, determina que a depuração da amônia está comprometida e confirma a acidose tubular distal. Outros estudos reportam resultados similares de acidoses distais usando métodos semelhantes com ou sem a necessidade de exames mais complexos - como a osmolaridade urinária - para confirmar o resultado do $\mathrm{AGU}^{32-35}$. Apesar dos benefícios, Batlle et al. ${ }^{36}$ alertam que seu resultado é apenas uma estimativa e deve ser observado com cuidado devido a quantidade de ânions não mensurados. Em vista disso, é necessário levar em conta a TFG para uma correção, logo, seu resultado só pode ser considerado fiel em pacientes que não estão com insuficiência renal aguda ou DRCs. Nesses casos, o cálculo do gap osmolar urinário se torna uma opção mais interessante. Na situação de um AGU duvidoso ou negativo, sugere-se o teste padrão ouro para confirmação de acidoses distais que é o teste do carregamento de amônia (ou carregamento de ácidos), onde o paciente ingere cloreto de amônio com a refeição (ou recebe o mesmo por infusão intravenosa na dosagem de $0,1 \mathrm{~g} / \mathrm{kg}$ 
corporal). Ainda existe uma terceira opção menos agressiva, onde há ingestão de $40 \mathrm{mg}$ de furosemida e $1 \mathrm{mg}$ de fludrocortisona, medicamentos que estimulam a secreção de íons de hidrogênio, então a cada 3 horas, é mensurado o $\mathrm{pH}$ sérico e urinário. Pessoas saudáveis conseguem eliminar o ácido plasmático, contudo, os que apresentarem $\mathrm{pH}$ urinário elevado não estão conseguindo eliminar o ácido presente no sangue, significando uma ATR distal ${ }^{36-37}$.

Notadamente, os cálculos utilizados para determinação de AG e AGU diferem pela presença - ou não - da concentração de bicarbonato (Tabela 3). Em urina relativamente ácida, a concentração de bicarbonato é essencialmente zero, e o seu efeito no $A G U$, portanto, insignificante ${ }^{16}$.

O AGU consegue confirmar rapidamente a presença da ATR eliminando a possibilidade de perda extrarrenal do bicarbonato, como problemas gastrointestinais ou em pacientes com acidose metabólica hiperclorêmica; e ainda consegue indicar se há uma possível ATR distal, desse modo, remove alguns obstáculos na busca do diagnóstico ${ }^{16,38}$. Para o diagnóstico de uma acidose proximal é necessário demonstrar a perda maior ou igual à $15 \%$ de $\mathrm{HCO}_{3}{ }^{-}$, e isso fica evidente quando o pH urinário está alto e a concentração de bicarbonato plasmático está normal ou um pouco baixo ${ }^{39,40}$. Há alta prevalência de acidose metabólica hiperclorêmica em pacientes em estado crítico, logo, AGU pode não ser a melhor alternativa devido à presença de ânions não medidos, como corpos cetônicos, por exemplo ${ }^{11}$.

Novos métodos de diagnóstico estão surgindo graças aos avanços da biologia molecular como a descoberta dos genes ATP6V1B1, ATP6V0A4 e SLC4A1 que codificam proteínas transportadoras de $\mathrm{H}^{+}$na porção distal dos túbulos. Apesar dos avanços, acredita-se que o método mais usado para o diagnóstico ainda será o laboratorial, pois exames moleculares são caros, pouco acessíveis e ainda em busca de otimização e padronização. Os exames laboratoriais são relativamente baratos e bastante acessíveis, sendo um ótimo exemplo o AGU, assim, a pesquisa molecular será importante para o aconselhamento genético de indivíduos afetados pelas mutações $^{41-42}$.

\section{CONCLUSÃO}

As ATRs são doenças extremamente complexas e pouco compreendidas, sendo sua principal característica, a acidose metabólica com ânion gap normalizado por hipercloremia. Podendo ser hereditárias ou adquiridas, apresentam um amplo espectro de sintomas oriundos da alteração no funcionamento de diversos mecanismos fisiológicos, sendo necessário formar uma linha de investigação para obtenção de resultado fidedigno em um tempo razoável, pois as consequências de uma acidose tubular renal podem ser severas, principalmente em crianças. Os avanços na biologia molecular proporcionam uma opção para o diagnóstico, porém, encontra-se ainda com grandes limitações para a rotina diagnóstica. O ânion gap urinário é, então, uma importante ferramenta nesta investigação, eliminando perdas extrarrenais de bicarbonato e diagnosticando a acidose tubular. O AGU é uma ferramenta de grande valia diagnóstica para as ATRs, já que necessita de informações de fácil acesso ou previamente obtidas, apresenta um baixo custo e há possibilidade de diagnóstico exato, desde que se leve em conta suas limitações (a fragilidade se acentua conforme há redução da taxa de filtração glomerular).

\section{REFERÊNCIAS BIBLIOGRÁFICAS}

1. National Kidney Foundation [homepage on the internet]. Global facts: about kidney disease [cited 2018 nov 03]. Available from: https://www.kidney.org.

2. World Health Organization. [homepage on the internet]. The global burden of kidney disease and the sustainable development goals [cited 2018 nov 02]. Available from: http://www.who.int.

3. Lozano R, Naghavi M, Foreman K, et. al. Global and regional mortality from 235 causes of death for 20 age groups in 1990 and 2010: a systematic analysis for the Global Burden of Disease Study 2010. The Lancet 2012;380(9859):2095-128.

4. Kraut JA, Nagami GT. Metabolic acidosis of chronic kidney disease. In: Ajay K. Singh A, Gordon H. Textbook of nephro-endocrinology. 2a edição. Cambridge: Academic Press; 2017. Capítulo 17.

5. Mustaqeem R, Arif A. Renal tubular acidosis. Treasure Island: StatPearls Publishing; 2019.

6. Fuster DG, Moe OW. Incomplete distal renal tubular acidosis and kidney stones. Advances in Chronic Kidney Disease 2018;25(4):366-374.

7. Guerra HN, Matos MM, Ordaz LKV, Muñiz MDC, Medeiros M, Pérez LE. Clinical and biochemical findings in Mexican patients with distal renal tubular acidosis. Rev Invest Clin 2014;66(5):386-92.

8. Seigle RL, Nash MA. Nephrology. In: Ditmar MF, Polin RA. Pediatric Secrets. 5a edição. Londres: Elsevier; 2015. Capítulo 13.

9. Sharma S, Gupta A, Saxena S. Comprehensive clinical approach to renal tubular acidosis. Clinical and Experimental Nephrology 2015;19(4):556-61.

10. Bello CHPRT, Duarte JS, Vasconcelos C. Diabetes mellitus and hyperkalemic renal tubular acidosis: case reports and literature review. J Bras Nefrol 2017;39(4):481-485.

11. Brunner R, Drolz A, Scherzer TM, Staufer K. Fuhrmann V, Zauner C, Holzinger U, Schneeweiß B. Renal tubular acidosis is highly prevalent in critically ill patients. Crit Care 2015;19:148.

12. Vallés PG, Batlle D. Hypokalemic distal renal tubular acidosis. Advances in Chronic Kidney Disease 2018;25(4):303-320.

13. Basu G, Sudhakar G, Mohapatra A. Renal tubular acidosis. Clin Quer Nephrol 2013;2:166-178.

14. Batlle D, Arruda J. Hyperkalemic forms of renal tubular acidosis: clinical and pathophysiological aspects. Advances in Chronic Kidney Disease 2018;25(4):321333.

15. Batlle D, Chen S, Haque SK. Physiologic principles in the clinical evaluation of electrolyte, water, and acidbase disorders. In: Alpern RJ, Hebert SC. Seldin and Giebisch's The Kidney. 5a edição. London: Academic Press; 2013. Capítulo 74.

16. Alexander RT, Bitzan M. Renal tubular acidosis. Pediatric Clinics of North America 2019;66:135-157.

17. Seifter JL. Acid-base disorders. In: Goldman L, Schafer A. Schafer Goldman's Cecil Medicine. 24a edição. Philadelphia: Saunders; 2012. Capítulo 120. 
18. Krapf R, Seldin DW, Alpern RJ. Clinical syndromes of metabolic acidosis. In: Alpern RJ, Hebert SC. Seldin and Giebisch's The Kidney. 5a edição. London: Academic Press; 2013. Capítulo 59.

19. Koeppen BM, Stanton BA. Regulation of acid-base balance. Renal Physiology. Philadelphia: Mosby; 2013.

20. Karet FE. Mechanisms in hyperkalemic renal tubular acidosis. Journal of the American Society of Nephrology 2009;20(2):251-4.

21. Nagami GT. Hyperchloremia - why and how. Nefrología 2016;36(4):347-53.

22. Berend K, Van Hulsteijn LH, Gans ROB. Chloride: the queen of electrolytes? European Journal of Internal Medicine 2012;23(3):203-11.

23. Shiber JR. Severe non-anion gap metabolic acidosis induced by topiramate: a case report. The Journal of Emergency Medicine 2010;38(4):494-6.

24. Riveiro-Barciela M, Campos-Varela I, Tovar JL, Vargas V, Simón-Talero $M$, Ventura-Cots $M$, Crespo M, Bilbao I, Castells L. Hyperkalemic distal renal tubular acidosis caused by immunosuppressant treatment with tacrolimus in a liver transplant patient: case report. Transplantation Proceedings 2011;43(10):4016-8.

25. Gil-Peña H, Mejía N, Santos F. Renal tubular acidosis. The Journal of Pediatrics 2014;164(4):691-698.

26. Kamel KS, Halperin ML, Goldstein MB. Fluid, Electrolyte and Acid-Base Physiology - A ProblemBased Approach. Philadelphia: Saunders; 2010.

27. Purohit S, Corry DB, Tuck ML. Hyporeninemic hypoaldosteronism. In: Huhtaniemi I, Martini L. Encyclopedia of Endocrine Diseases. 2a edição. Massachussetts: Academic Press; 2018.

28. Landry DW, Bazari H. Approach to the patient with renal disease. In: Goldman L, Schafer A. Schafer Goldman's Cecil Medicine. 24a edição. Philadelphia: Saunders; 2012. Capítulo 116.

29. Haque SK, Ariceta G, Batlle D. Proximal renal tubular acidosis: a not so rare disorder of multiple etiologies. Nephrol Dial Transplant 2012;27(12):4273-87.

30. Yaxley J, Pirrone C. Review of the diagnostic evaluation of renal tubular acidosis. Ochsner J 2016;16(4):525530.

31. Soleimani M, Rastegar A. Pathophysiology of renal tubular acidosis: core curriculum 2016. American Journal of Kidney Diseases 2016;68(3):488-98.

32. Sarma A. Hypokalemic paralysis due to primary Sjogren syndrome. Indian Journal of Endocrinology and Metabolism 2018;22(2):287-289.

33. Van Den Wildenberg MJ, Hoorn EJ, Mohebbi N, Wagner CA, Woittiez AJ, de Vries PA, Laverman GD. Distal renal tubular acidosis with multiorgan autoimmunity: a case report. American Journal of Kidney Diseases 2015;65(4):607-10.

34. Muthukrishnan J, Dawra S, Marwaha V, Narayanan CS. Sjögren's syndrome presenting as hypokalemic paralysis. Medical Journal Armed Forces India 2015l;71(Suppl 1):S172-S174.

35. Zhang C, Ren H, Shen P, Xu Y, Zhang W, Wang W, Li X, Ma $Y$, Chen N. Clinical evaluation of chinese patients with primary distal renal tubular acidosis. Internal Medicine 2015;54(7):725-30.

36. Batlle $\mathrm{D}, \mathrm{Ba}$ Aqeel $\mathrm{SH}$, Marquez $\mathrm{A}$. The urine anion gap in context. Clinical Journal of the American Society of Nephrology 2018;13(2):195-197.

37. Both T, Zietse R, Hoorn EJ, van Hagen PM, Dalm VA, van Laar JA, van Daele PL. Everything you need to know about distal renal tubular acidosis in autoimmune disease. Rheumatology International 2014;34(8):1037-45.

38. Finer G, Landau D. Clinical approach to proximal renal tubular acidosis in children. Advances in Chronic Kidney Disease 2018;25(4):351-357.

39. Syed KH, Ariceta G, Batlle D. Proximal renal tubular acidosis: a not so rare disorder of multiple etiologies. Nephrol Dial Transplant 2012;27(12):4273-87.

40. Oliveira JR, Lunardelli A. Equilíbrio Ácido-básico. In: Oliveira JR. Alterações clínicas e laboratoriais do metabolismo iônico. Porto Alegre: Edipucrs; 2011. Capítulo 8.

41. Boualla L, Jdioui W, Soulami K, Ratbi I, Sefiani A. Clinical and molecular findings in three Moroccan families with distal renal tubular acidosis and deafness: report of a novel mutation of ATP6V1B1 gene. Current Research in Translational Medicine 2016;64(1):5-8.

42. Magen $D$, Zelikovic I. Hereditary tubular disorders of mineral handling. In: Glorieux FH, Pettifor JM, Jüppner H. Pediatric Bone. 2a edição. London: Academic Press; 2012. Capítulo 27. 